\title{
INMOVILIZACIÓN DE LA GÜIÑA (LEOPARDUS GUIGNA) EN ESTADO SILVESTRE CON LA ASOCIACIÓN ANESTÉSICA KETAMINA-XILACINA
}

\author{
IMMOBILIZATION OF KODKOD (LEOPARDUS GUIGNA) WITH KETAMINE \\ HYDROCHLORIDE AND XYLAZINE HYDROCHLORIDE
}

\author{
Gerardo Acosta J. M.V., M.S., Ph.D. (C), ${ }^{1}$ Stephan M. Funk, Ph.D. ${ }^{2}$ \\ AND Nigel Dunstone, PH.D. ${ }^{3}$
}

\begin{abstract}
The kodkod (Leopardus guigna) is one of the least known endangered felid species. In order to find an adequate anaesthetic association to handle safely kodkod individuals during ecological studies and veterinary procedures, we conducted 10 chemical immobilizations in 7 animals using a combination of ketamine hydrochloride (KH) and xylazine hydrochloride (XH) at the Laguna San Rafael National Park (Chile). A mean ( $\pm S E$ ) dosage of $15.4 \pm 3.2 \mathrm{mg} / \mathrm{kg} \mathrm{KH}$ and $1.7 \pm 0.3 \mathrm{mg} / \mathrm{kg} X H$ resulted in mean induction time of $4.6 \pm 2.9 \mathrm{~min}$ and a mean recovery time of $63.9 \pm 31.9 \mathrm{~min}$. There was a direct relationship between $\mathrm{KH}$ dosage and the duration of the immobilization, with the combination of drugs leading to a shorter induction time. No secondary effects were observed. Based on our preliminary results, we recommended dosages of $11-12 \mathrm{mg} / \mathrm{kg} \mathrm{KH}$ and $1.6-1.7 \mathrm{mg} / \mathrm{kg} \mathrm{XH}$.
\end{abstract}

\section{INTRODUCCIÓN}

La inmovilización química de los animales silvestres a menudo es necesaria con fines de investigación, de manejo y para la realización de tratamientos veterinarios (Johnson y Franklin, 1991; Adams, 2001; De Leeuw y col., 2004). Para realizar una inmovilización segura es importante utilizar drogas anestésicas que provean el efecto adecuado. La ketamina es uno de los anestésicos más ampliamente utilizados en el estudio de mamíferos silvestres, particularmente en carnívoros, tanto sola como en combinación con otras drogas. Es particularmente utilizada y se recomienda su uso para la inmovilización de felinos silvestres (Harthoorn, 1973; Ramsden y col., 1976; Fowler, 1986; Logan y col., 1986; Kreeger y col., 1990; Ferreras y col., 1994). Es un anestésico disociativo, que produce anestesia cataléptica con analgesia

Institute of Zoology, Zoological Society of London, Regent's Park, London, NW1 4RY, UK.

2 Nature Heritage Ltd, London, UK.

3 Centre for Tropical Ecology, School of Biological and Biomedical Sciences, University of Durham, South Road, Durham City, DH1 3LE, UK. moderada (Burroughs, 1993). Luego de su inyección es rápidamente absorbida y distribuida en todos los tejidos corporales, principalmente en el tejido adiposo, hígado, pulmones y cerebro (Adams, 2001). Sus principales ventajas son el amplio margen de seguridad, bajo costo y amplia disponibilidad; entre sus desventajas se encuentran la excesiva salivación que produce, contracción muscular y midriasis (Adams, 2001). La combinación de ketamina con tranquilizantes, como por ejemplo xilacina, ha sido utilizada para mejorar la anestesia y reducir algunos efectos colaterales no deseados, especialmente excesiva contracción muscular y catalepsia (Knight, 1980). La xilacina es un agonista $\alpha-2$ adrenérgico que produce una potente depresión del sistema nervioso central, lo cual lleva a la sedación (Knight, 1980; Morin y Berteaux, 2003).

Existe una tendencia en desarrollar y evaluar técnicas de inmovilización que permitan a los investigadores manipular con seguridad a los animales silvestres en su medio natural (Telesco y Sovada, 2002). Cualquier procedimiento veterinario que se realice en animales salvajes puede causar estrés, lo cual puede incrementar la posibilidad de desarrollar enfermedades (Adams, 2001). Por lo tanto, es 
necesario que cualquier procedimiento anestésico a desarrollar sea evaluado y optimizado, con el fin de producir una inducción corta, una óptima duración de la inmovilización según el procedimiento a realizar y una adecuada relajación muscular (Telesco y Sovada, 2002). En este artículo evaluamos la inmovilización de la güiña (Leopardus guigna) en estado silvestre, utilizando la asociación anestésica ketamina-xilacina, con el fin de manipular animales que fueron capturados para marcaje, instalación de radiocollares y evaluación de su estado de salud.

La güiña es el felino silvestre más pequeño de América y uno de los félidos en peligro de extinción menos conocidos en el mundo (Nowell y Jackson, 1996; IUCN, 2002). La investigación se llevó a cabo como parte de un proyecto de investigación sobre la ecología de la especie en el Parque Nacional Laguna San Rafael, XI Región de Chile (Dunstone $y$ col., 2002). Restricciones de orden logístico en esta remota área no nos permitieron un monitoreo más intensivo de los animales inmovilizados. El objetivo de este estudio fue determinar una dosis apropiada para manipular con seguridad a los animales por aproximadamente 30 minutos, tiempo suficiente para realizar las actividades planificadas y posteriormente liberarlos. Las dosis fueron evaluadas mediante el monitoreo de tres parámetros básicos: tiempo de inducción (TI), tiempo de recuperación (TR) y relajación muscular (RM).

\section{MATERIALES Y MÉTODOS}

El trabajo de campo se realizó en el Parque Nacional Laguna San Rafael, XI Región de Chile (46 38'S; $73^{\circ} 51^{\prime} \mathrm{W}$ ). El clima es templado marino frío con una alta humedad y un promedio de precipitación anual de $3.700 \mathrm{~mm}$ y un promedio de temperatura durante el verano de $11,3^{\circ} \mathrm{C}$. Durante enero-marzo de 1998 se instalaron nueve trampas-caja $(105 \mathrm{~cm}$ x $50 \mathrm{~cm}$ x 37,5 cm, Tomahawk Live Trap Company, Wisconsin) en sitios seleccionados y se revisaron dos veces al día según se describe en Dunstone $y$ col. (2002). El éxito durante este periodo fue de 20 capturas en 468 días de trampeo. En 10 capturas los animales fueron liberados inmediatamente $\sin$ manipulación debido a que eran recapturas. Se realizaron 10 inmovilizaciones químicas en 7 animales diferentes. Dos machos adultos y una hembra adulta fueron inmovilizados dos veces dentro de 25, 20 y 19 días, respectivamente, analizándose estos datos de manera independiente (Seal y col., 1987; Beltrán y
Tewes, 1995). Con la finalidad de reducir el trauma al que eran sometidos los animales durante la inmovilización, se utilizó un panel apretador dentro de la trampa para restringir el movimiento de los gatos en el momento de la inyección de la combinación anestésica. Ketamina (Ketaset, Parke, Davis \& Co., Detroit, Mitch.) y xilacina (Rompum, Bayer) se mezclaron inmediatamente previa a la inyección en una jeringa desechable de tuberculina. Las dosis de los fármacos ( $\mathrm{mg} / \mathrm{kg}$ de peso muscular) se calcularon inicialmente para ser utilizadas en un gato de peso promedio de 2,0 $\mathrm{kg}$ según lo reportado en la literatura (Greer, 1965). Después de haber realizado algunas capturas se observó que el peso real de los animales era menor al peso reportado en la literatura, por lo cual las dosis fueron ajustándose paulatinamente a un peso corporal promedio de $1,5 \mathrm{~kg}$, por lo cual las dosis finales variaron por animal dependiendo del peso real obtenido. Además, la razón ketamina/xilacina $(\mathrm{K} / \mathrm{X})$ -la cual es una expresión de la proporción de mg de ketamina por cada mg de xilacina utilizado y entrega una idea de los efectos de la ketamina y cómo éstos son contrarrestados por la xilacina utilizada- también fue ajustándose dependiendo de la respuesta encontrada en los animales (i.e., relajación muscular adecuada, profundidad de anestesia, entre otros. Ver más abajo). Durante la anestesia los individuos fueron marcados con autocrotales, se les tomaron medidas morfométricas, se les instalaron radiocollares y se les tomó una muestra de tejido desde la oreja para realizar análisis genéticos. El peso corporal de los animales se determinó mediante el pesaje de los individuos con una balanza manual ( $\pm 0,1 \mathrm{~kg}$, Pesola). Para prevenir que los animales entraran en hipotermia, éstos fueron cubiertos con una frazada térmica. Para minimizar el estrés durante el procedimiento se evitó realizar ruidos innecesarios y las orejas y los ojos de los animales fueron cubiertos para evitar los estímulos auditivos y luminosos, respectivamente. El tiempo de inducción (TI) se definió como el intervalo entre la inyección de los fármacos hasta la inmovilización y falta de respuesta del animal. El tiempo de recuperación (TR) correspondió al tiempo desde el inicio de la inmovilización (inducción) hasta cuando se detectó el primer movimiento de la cabeza. El grado de relajación muscular (RM) fue evaluado mediante la suave tracción de los miembros posteriores cada 5 minutos y de esta forma se determinó la tensión muscular en una escala del 1 al 4, según: animal totalmente tenso (1), escasa (2), moderada (3) y excelente (4) relajación muscular, no habiendo en esta última dificultad alguna en traccionar los 
miembros del animal. La temperatura corporal y la frecuencia respiratoria fueron medidas luego de 10 minutos de la inducción. La frecuencia cardiaca fue medida sólo en cinco ocasiones luego de 20 minutos de la inducción. Antes o inmediatamente después del primer movimiento de la cabeza, los animales fueron puestos de nuevo dentro de la trampa, donde permanecieron hasta su recuperación total de los efectos de la anestesia (por ejemplo, cuando ningún signo aparente de incoordinación fuese evidente).

Se utilizó la prueba de U de Mann-Whitney (Zar, 1984) para analizar si existía alguna diferencia entre sexos en relación de las dosis de ketamina y xilacina ( $\mathrm{mg} / \mathrm{kg}$ de peso corporal) como variables independientes y TI y TR como variables dependientes. Regresiones simples y múltiples se utilizaron para determinar la influencia de las dosis de los fármacos utilizados en el TI y TR. Finalmente, se utilizó la prueba no-paramétrica de correlación de Spearman para analizar la relación entre la razón ketamina/xilacina $(\mathrm{K} / \mathrm{X})$ con el grado de relajación muscular obtenido. Todos los niveles de significancia se ajustaron en $\mathrm{p}<0,05$.

\section{RESULTADOS Y DISCUSIÓN}

En total 4 machos adultos, dos hembras adultas y una cría macho fueron capturados. El promedio $( \pm \mathrm{ES})$ del peso corporal fue $1,52 \pm 0,2 \mathrm{~kg}$ sin ninguna diferencia evidente por sexos (Tabla 1). El promedio de las dosis de ketamina y xilacina utilizadas fue de $15,4 \pm 3,2 \mathrm{mg} / \mathrm{kg}$ y $1,7 \pm 0,3 \mathrm{mg} / \mathrm{kg}$, respectivamente. El promedio del tiempo de recuperación, TR, fue de $63,9 \pm 31,9$ minutos, con un promedio de $\mathrm{TR}=77,8$ $\pm 26,2$ minutos para los machos y un promedio de $\mathrm{TR}=36 \pm 24,6$ minutos en las hembras. El promedio de tiempo de inducción, TI, fue 4,6 \pm 2.9 minutos, con un promedio de $\mathrm{TI}=4,0 \pm 2,8$ minutos en los machos $\mathrm{y}$ un promedio de $\mathrm{TI}=5,8 \pm 3,3$ minutos en las hembras. Se capturó sólo una cría que mostró un TI de $5 \mathrm{~min}$ y un TR de 24 min (Tabla 1). No hubo diferencias significativas entre las dosis de ketamina $(Z=1,81$, $\mathrm{p}=0,07)$, xilacina $(Z=0,9, \mathrm{p}=0,37)$ ni la razón $\mathrm{K} / \mathrm{X}$ $(Z=1,67, \mathrm{p}=0,09)$ ni entre el RT $(Z=1,56, \mathrm{p}=0,12) \mathrm{e}$ IT $(Z=0,77, p=0,48)$ entre sexos. Esto es contrario a lo encontrado por Beltrán y Tewes (1995), quienes reportaron menores TI en machos que en hembras linces y ocelotes, no obstante es importante tener en cuenta que nuestro tamaño de muestra separado por sexo fue considerablemente pequeño, por lo cual estos resultados deben tomarse en cuenta con cierta precaución.

$\mathrm{Al}$ investigarse el efecto de las dosis de los fármacos utilizadas con la razón K/X, el TI y el TR se encontró que la dosis de ketamina fue positivamente correlacionada con el TR $\left(r_{\mathrm{s}}=0,77, \mathrm{p}=0,009\right)$, pero no con el TI $\left(\mathrm{r}_{\mathrm{s}}=-0,39, \mathrm{p}=0,27\right)$. No hubo correlación significativa entre la dosis de xilacina y el TR $\left(r_{\mathrm{s}}=-0,1, \mathrm{p}=0,79\right)$ ni el TI $\left(\mathrm{r}_{\mathrm{s}}=-0.5, \mathrm{p}=0,15\right)$. Un análisis de regresión múltiple mostró una correlación positiva al analizar en forma conjunta las dosis de ketamina y xilacina con el TR $(R=0,82, p=0,02)$. Esta relación fue mejor explicada por el efecto de la ketamina $(\beta=0,92$, $\mathrm{p}=0,007)$ más que el efecto de la xilacina $(\beta=0,32$, $\mathrm{p}=0,22$ ). Además, este análisis indicó una significativa correlación positiva entre las dosis de ketamina y xilacina y el TI $(\mathrm{R}=0,85, \mathrm{p}=0,01)$, contribuyendo en este caco ambas drogas a esta asociación $(\mathrm{K}: \beta=-0,77$, $p=0,01 ; X: \beta=-0,85, p=0,007)$. Se encontró una fuerte correlación negativa entre la razón $\mathrm{K} / \mathrm{X}$ y el grado de relajación muscular $\left(\mathrm{r}_{\mathrm{s}}=-0,83, \mathrm{p}=0,04\right)$. El grado de relajación muscular alcanzó su máximo valor (4) bajo una razón $\mathrm{KH} / \mathrm{XH}$ de $8: 1$.

TABLA 1

RESUMEN DE DOSIS Y RESPUESTAS FISIOLÓGICAS DE LA GÜIÑA (LEOPARDUS GUIGNA) CON LA ASOCIACIÓN ANESTÉSICA KETAMINA-XILACINA EN PARQUE NACIONAL LAGUNA SAN RAFAEL, XI REGIÓN CHILE ${ }^{1}$

\begin{tabular}{|c|c|c|c|c|c|c|c|c|c|}
\hline Categoría & $\begin{array}{c}N^{\circ} \text { de } \\
\text { animales }\end{array}$ & $\begin{array}{c}\text { Peso } \\
\text { (kg) }\end{array}$ & $\begin{array}{c}N^{\circ} \text { de } \\
\text { procedimientos }\end{array}$ & $\begin{array}{l}\text { Ketamina } \\
(\mathrm{mg} / \mathrm{kg})\end{array}$ & $\begin{array}{c}\text { Xilacina } \\
(\mathrm{mg} / \mathrm{kg})\end{array}$ & $\begin{array}{c}\text { Razón } \\
K / X\end{array}$ & $\begin{array}{c}T I \\
(\min )\end{array}$ & $\begin{array}{c}T R \\
(\min )\end{array}$ & $G R M^{2}$ \\
\hline Adultos & 6 & $1,52 \pm 0,2$ & 9 & $15,4 \pm 3,2$ & $1,7 \pm 0,3$ & $10,0 \pm 4,0$ & $4,6 \pm 2,9$ & $63,9 \pm 31,9$ & $4(1-4)$ \\
\hline Machos & 4 & $1,58 \pm 0,2$ & 6 & $16,7 \pm 2,9$ & $1,6 \pm 0,4$ & $11,4 \pm 4,3$ & $4,0 \pm 2,8$ & $77,8 \pm 26,2$ & $3(1-4)$ \\
\hline Hembras & 2 & $1,42 \pm 0,1$ & 3 & $12,9 \pm 2,2$ & $1,8 \pm 0,2$ & $7,1 \pm 0,6$ & $5,8 \pm 3,3$ & $36,0 \pm 24,6$ & $4(4-4)$ \\
\hline Crías & 1 & 0,85 & 1 & 11,8 & 1,65 & 7,15 & 5 & 24 & 4 \\
\hline
\end{tabular}

1 Todos los valores están en promedio \pm ES, excepto para caso de animales, procedimientos y para el grado de relajación muscular (mediana y rango).

${ }^{2}$ GRM: grado de relajación muscular. 
La temperatura luego de 10 minutos de inducción fue de $37,6 \pm 0,8 \mathrm{C}$ (rango: $36,7-38.9 ; \mathrm{n}=9$ ), sin diferencias entre sexos $(t=1,03, \mathrm{P}=0,34)$. La frecuencia respiratoria fue de $26 \pm 2$ ciclos por minuto (rango 22-30; $\mathrm{n}=9)$, sin diferencias entre $\operatorname{sexos}(t=0,87$, $\mathrm{P}=0,41)$. Finalmente, la frecuencia cardiaca luego de 20 minutos de inducción fue de $132 \pm 10$ latidos por minuto (rango 130-150; $\mathrm{n}=5$ ).

En las primeras capturas realizadas utilizamos las dosis de referencia publicadas en la literatura para felinos pequeños (i.e., $15 \mathrm{mg} / \mathrm{kg} \mathrm{K} \mathrm{y} 2 \mathrm{mg} / \mathrm{kg} \mathrm{X}$ ) (Logan y col., 1986; Beltrán y Tewes, 1995), utilizando un peso estimado de $2 \mathrm{~kg}$. Posteriormente, las dosis se fueron reduciendo progresivamente manteniendo una razón $\mathrm{K} / \mathrm{X}$ alrededor de 6:1-8:1 y un óptimo grado (i.e., 4) de relajación muscular. Esta modificación se realizó hasta lograr un TR de alrededor de 30 minutos, lo cual era un tiempo suficiente para realizar las actividades necesarias mientras los animales se encontraban inmovilizados (e.g., retiro o instalación de radiocollar, toma de muestras). Sanderson y col. (2002) capturaron ocho güiñas en la Isla de Chiloé, X Región, Chile, y reportaron una dosis de $20 \mathrm{mg}$ de ketamina por kg de peso corporal, pero no indicaron ningún resultado de la anestesia realizada, por lo cual no se pueden hacer comparaciones con el procedimiento que ellos realizaron.

Nuestro tamaño de muestra es indiscutiblemente pequeño debido al escaso éxito de captura, el cual fue severamente restringido debido a dificultades logísticas y de infraestructura. Sin embargo, nuestras observaciones confirman que la combinación ketamina-xilacina puede ser utilizada exitosamente en güiñas. Similares TI y TR han sido observados en otras especies de felinos. Por ejemplo, linces y ocelotes fueron inmovilizados con 13,3 y 14,7 $\mathrm{mg} / \mathrm{kg}$ de ketamina y 1,2 y $1,1 \mathrm{mg} / \mathrm{kg}$ de xilacina, respectivamente (Beltrán y Tewes, 1995). Con esas dosis ellos reportaron un TI promedio entre 9-12 min y un TR entre 40-50 min, para linces y ocelotes, respectivamente. Logan y col. (1986) reportaron una dosis promedio de $11 \mathrm{mg} / \mathrm{kg}$ de ketamina y $1,8 \mathrm{mg} / \mathrm{kg}$ de xilacina con un TI promedio de 6 min y un TR de 50 min. Fuller $y$ col. (1985) usaron una dosis de $11 \mathrm{mg} / \mathrm{kg}$ de ketamina y $1,5 \mathrm{mg} / \mathrm{kg}$ de xilacina para inmovilizar linces. Esta combinación no produjo la muerte de ningún animal ni tampoco ningún efecto secundario como por ejemplo convulsiones. Las dosis utilizadas por Fuller $y$ col. (1985) se asemejan a las dosis que finalmente utilizamos en nuestro estudio y que presentaron una buena relajación muscular, corta inducción y un tiempo de recuperación de entre 30-
40 minutos. Sin embargo, debido a nuestro pequeño tamaño de muestra es importante tener en cuenta que los efectos secundarios ocurren en bajo porcentaje, por lo que al aumentar el tamaño muestral también podría aumentar el riesgo de que ocurran efectos indeseados. De hecho, Grassmann Jr. y col. (2004) reportaron la ocurrencia de convulsiones en un $2 \%$ de leopardos inmovilizados con una razón $\mathrm{K} / \mathrm{X}=14$, y una dosis promedio de ketamina y xilacina de 27,4 y $1,9 \mathrm{mg} / \mathrm{kg}$, respectivamente. También, Ferreras y col. (1994), reportaron un $9 \%$ de convulsiones en lince Ibérico con una razón $\mathrm{K} / \mathrm{X}=0,92$, y un promedio de dosis de ketamina y xilacina de 4,7 y $5,1 \mathrm{mg} / \mathrm{kg}$, respectivamente. Es posible que las convulsiones reportadas en los dos estudios anteriores se hayan debido en el primer caso a una excesiva dosis de ketamina utilizada y en el segundo caso a una excesiva dosis de xilacina, la cual se ha descrito que produce convulsiones en altas concentraciones (Knight, 1980). El uso de esta asociación parece producir una baja mortalidad. En puercoespines se ha reportado una mortalidad de sólo un $0,87 \%$ (Morin y Berteaux, 2003), y así como ha ocurrido en el presente trabajo varios estudios en felinos no han reportado la ocurrencia de mortalidad tal como se ha reportado en puma (Logan y col., 1986); lince ibérico (Ferreras y col., 1994) y ocelotes y linces (Beltrán y Tewes, 1995). Esto reconfirma el hecho del amplio margen de seguridad de esta combinación (Gregg y Olson, 1975).

La asociación anestésica utilizada en este estudio ha sido exitosamente usada por más de 30 años en la contención de fauna silvestre. A pesar de las desventajas que esta asociación posee (e.g., dificultad de reversión y convulsiones) comparada con otras drogas y asociaciones más modernas, es bastante segura como se confirma aquí para su uso en gato güiña, sumado al hecho de su amplia disponibilidad y bajo costo en el país (Fowler, 1986; Adams, 2001). A pesar del tamaño de muestra, nuestras observaciones dan sustento al uso seguro de la combinación ketamina-xilacina. Nosotros recomendamos una dosis de ketamina de entre $11-12 \mathrm{mg} / \mathrm{kg}$ y $1,6-1,7$ $\mathrm{mg} / \mathrm{kg}$ de xilacina para permitir la manipulación de güiñas en su estado silvestre durante periodos de aproximadamente 30 minutos.

\section{AGRADECIMIENTOS}

Agradecemos el financiamiento entregado a este proyecto por parte de la Darwin Initiative, the People's Trust for Endangered Species, y the Ernest Kleinwort 
Charitable Trust. También agradecemos especialmente a la Corporación Nacional Forestal, XI Región, por todo el apoyo logístico prestado y por permitirnos el acceso al área de estudio bajo su jurisdicción y poder así llevar a cabo este estudio.

\section{RESUMEN}

La güiña (Leopardus guigna) es uno de los felinos silvestres en peligro de extinción menos conocidos. Con el fin de encontrar una asociación anestésica adecuada para la manipulación segura de güiñas durante procedimientos veterinarios y estudios ecológicos nosotros realizamos 10 inmovilizaciones químicas en 7 animales diferentes utilizando una combinación de ketamina y xilacina en el Parque Nacional Laguna San Rafael, XI región, Chile. En promedio $( \pm \mathrm{ES})$ las dosis de $15,4 \pm 3,2 \mathrm{mg} / \mathrm{kg}$ de ketamina y $1,7 \pm 0,3 \mathrm{mg} / \mathrm{kg}$ de xilacina produjeron un tiempo de inducción promedio de 4,6 $\pm 2,9$ min y un tiempo de recuperación de 63,9 $9 \pm 31,9$ min. Hubo una directa correlación entre las dosis de ketamina y la duración de la inmovilización, con la combinación de ambas drogas llevando a un menor tiempo de inducción. No se observaron efectos secundarios. Basados en nuestros resultados recomendamos dosis entre 11-12 $\mathrm{mg} / \mathrm{kg}$ de ketamina y $1,6-1,7 \mathrm{mg} / \mathrm{kg}$ de xilacina para realizar procedimientos de corta duración en güiñas en su estado silvestre.

\section{REFERENCIAS}

ADAMS, H.R. 2001. Ed. Veterinary Pharmacology and Therapeutics. Ames, Iowa State University Press.

BELTRÁN, J.F. y M.E. TEWES. 1995. Immobilization of ocelots and bobcats with ketamine hydrochloride and xylazine hydrochloride. J. of Wild. Dis. 31:43-48.

BURROUGHS, R.E.J. 1993. A summary of the practical aspects of drugs commonly used for the restraint of wild animals. The Capture and Care Manual. Pretoria, South Africa, Wildlife Decision Support Services and the South African Veterinary Foundation: 65-70. A. E. MCKENZIE.

DE LEEUW, A.N.S.; G.J. ForRESTER; P.D. SPYVEE; M.G.I. BRASH y R.J. DELAHAY. 2004. Experimental comparison of ketamine with a combination of ketamine, butorphanol and medetomidine for general anaesthesia of the Eurasian badger (Meles meles L.). The Veterinary Journal 167:186-193.

Dunstone, N.; L. Durbin; I. Wyllie; R. Freer; G.A. ACosta; M. MAZZOLLI y S. RosE. 2002. Spatial organization, ranging behaviour and habitat utilization of the Leopardus guigna in southern Chile. J. of Zool. (London) 257:1-11.

Ferreras, P.; J.J. Aldama; J.F. Beltrán y M. Delibes. 1994. Immobilization of the endangered Iberian lynx with xylazine- and ketamine hydrochloride. J. of Wild. Dis. 30:63-68.

FowLER, M.E. 1986. Ed. Zoo and Wild Animal Medicine. Philadelphia, W.B. Saunders Co.

Fuller, T.K.; K.D. KeER y P.D. KARNS. 1985. Hematology ad serum chemistry of bobcats in north central Minesota. J. of Wild. Dis. 21:29-32.

GRASSMAN JR., L.I.; S.C. AUSTIN; M.E. TEWES y N.J. SILVY. 2004. Comparative Immobilization of Wild Felids in Thailand. J. of Wild. Dis. 40:575-578.

Greer, J.K. 1965. Mammals of Malleco Province, Chile. Publ. Mich. State Univ. B. 3:49-152.

GrEGG, D.A. y L.D. Olson. 1975. The use of ketamine hydrochloride as an anesthesic for racoons. J. of Wild. Dis. 11:335-337.

HARTHOORN, A.M. 1973. Reviews of wildlife capture drugs in common use. The Capture and Care of Wild Animals. Cape Town, Human \& Roisseaux: 14-34. E. YouNG.

IUCN. 2002. IUCN Red List of Threatened Species. Gland, Switzerland, International Union for Conservation of Nature and Resource.

Johnson, W.E. y W.L. FrankLin. 1991. Feeding and spatial ecology of Felis geoffroyi in southern patagonia. J. of Mammal. 72:815-820.

KNIGHT, A.P. 1980. Xylazine. JAVMA 176:454-455.

Kreeger, T.J.; U.S. SeAl y J.R. TeSter. 1990. Chemical immobilization of red foxes (Vulpes vulpes). J. of Wild. Dis. 26:95-98.

LOGAN, K.A.; E.T. THORNE; L.L. IRWIN y R. SKINNER. 1986. Immobilizing Wild Mountain Lions (Felis concolor) with Ketamine Hydrochloride and Xylazine Hydrochloride. J. of Wild. Dis. 22(1):97-103.

Morin, P. y D. BERTEAUX. 2003. Immobilization of North American porcupines (Erethizon dorsatum) using ketamine and xylazine. J. of Wild. Dis. 39:675-682.

Nowell, K. y P. JACKSON. 1996. Wild cats. Status survey and conservation plan. Gland, Switzerland, International Union for Conservation of Nature and Resource.

RAMSDEN, R.O.; P. F. COPPIN y D.H. JOHNSTON. 1976. Clinical observation on the use of ketamine hydrochloride in wild carnivores. J. of Wild. Dis. 12:221-225.

SANDERSON, J.; M.E. SUNQUIST y A. IRIARTE. 2002. Natural history and Landscape-use of guignas (Leopardus guigna) on Isla Grande de Chiloé, Chile. J. of Mammal. 83:608-613.

SEAL, U.S.; D. L. ARMSTRONG y L.G. SimMONS. 1987. Yohimbine hydrochloride reversal of ketamine hydrochloride and xylazine hydrochloride immobilization of Bengal tigers and effects on haematology and serum chemistries. J. of Wild. Dis. 23:296-300.

TELESCO, R.L. y M.A. SOVADA. 2002. Immobilization of swift foxes with ketamine hydrochloride-xylazine hydrochloride. J. of Wild. Dis. 38:764-768.

ZAR, J.H. 1984. Biostatistical analysis. London, England, Prentice-Hall International (UK) Limited. 